\title{
Expiration Training System for Treatment of People with Speech Problems
}

\author{
Yasuhiro Ogoshi*, Sakiko Ogoshi ${ }^{1}$, Kyoko Yamazaki², Tomohiro Takezawa ${ }^{3}$, \\ Akio Nakai ${ }^{4,5}$, Kiyoharu Tsuji ${ }^{6}$ and Yoshinori Mitsuhashi ${ }^{7}$ \\ Graduate School of Engineering, University of Fukui, 3-9-1 Bunkyo, Fukui 910-8507, Japan \\ ${ }^{1}$ Career Design Laboratory for Gender Equality, Kanazawa University, \\ Kakuma-machi, Kanazawa 920-1192, Japan \\ ${ }^{2}$ Fukui College of Health Sciences, 55-13-1 Egamicho, Fukui 910-3190, Japan \\ ${ }^{3}$ The National Institute of Vocational Rehabilitation, \\ 3-1-3 Wakaba, Mihama-ku, Chiba 261-0014, Japan \\ ${ }^{4}$ Hyogo Children's Sleep and Development Medical Research Center, \\ 1070 Akebono-cho, Nishi-ku, Kobe 651-2181, Japan \\ ${ }^{5}$ Department of Child and Adolescent Psychological Medicine, University of Fukui Hospital, \\ Matsuoka-shimoaizuki, Eiheiji-cho, Yoshida-gun, Fukui 910-1193, Japan \\ ${ }^{6}$ Fukui Prefectural Rehabilitation Center for Children with Disabilities, \\ 2-8-1 Yotsui, Fukui 910-0846, Japan \\ ${ }^{7}$ Faculty of Education and Regional Studies, University of Fukui, \\ 3-9-1 Bunkyo, Fukui 910-8507, Japan
}

(Received January 14, 2014; accepted April 30, 2014)

Key word: speech therapist, motor speech disorders, articulation disorders, rehabilitation, expiration training, air flow sensor

For a speech therapist, the breathing method is one important technique for training people with speaking difficulties. In the past, a trainer used the so-called "blowing technique" or "breathing play" for speech-related expiration training. However, insufficient numbers of therapists make it difficult to widely apply these breath-training methods, and the development of new breath training methods has become mandatory. Therefore, a new training system, in which the expiration pattern of a speech therapist is analyzed using an airflow sensor and converted into a graphic visual image to facilitate the easy recognition of expiration patterns, was developed. Experimentally, using this system with nonhandicapped trainees demonstrated that such trainees could successfully and easily imitate the trainer's pronunciation. It is suggested that people with speech problems would be able to easily learn appropriate speech-related expiration patterns by using the visual patterns produced by this system.

${ }^{*}$ Corresponding author: e-mail: y-ogoshi@u-fukui.ac.jp 


\section{Introduction}

Various training methods have been implemented by speech therapists for people with speech, language and hearing disorders. ${ }^{(1-4)}$ Among them, expiration training is considered highly important for improving motor functions of articulatory organs. One expiration training method is the "blowing technique", in which a trainee blows air through a straw placed at a depth of $5-10 \mathrm{~cm}$ in a cup of water. The expiration power necessary for making an utterance $\left(5-10 \mathrm{~cm} \mathrm{H}_{2} \mathrm{O}\right)$ and duration $(5 \mathrm{~s})$ are verified, and then the strength of respiratory muscles is increased accordingly.

Various pronunciation techniques giving visual feedback of breath control have been conducted for pronunciation training. These include blowing at a candle (rhythmically, alternating between strong and weak blows, so as not to put out the candle) and blowing feather flutes, party horns, paper balloons, blowing balls, ping-pong balls, and pinwheels, among others. ${ }^{(7)}$ For vocalizing continuous sounds (smoothly shifting from one single sound to another), blowing that can be visually confirmed is considered effective ${ }^{(8)}$ because continuous long expirations are required.

We have developed a system in which trainees can imitate therapists' expiration patterns (e.g., timing, strength, and length of expiration) by visually examining the patterns in a graph shown through a computer. In this method, a trainee holds a mouthpiece with a chosen shape and simultaneously watches a graph indicating his/her expiration patterns. ${ }^{(9,10)}$ It was confirmed that training using this system was effective for decreasing the differences in expiration patterns between a therapist and a trainee, which were calculated from the graph.

\section{Suggested System}

\subsection{Structure of system}

The structure of the system is shown in Fig. 1, expiration training reported in this paper was conducted with this system. The system includes various elements such

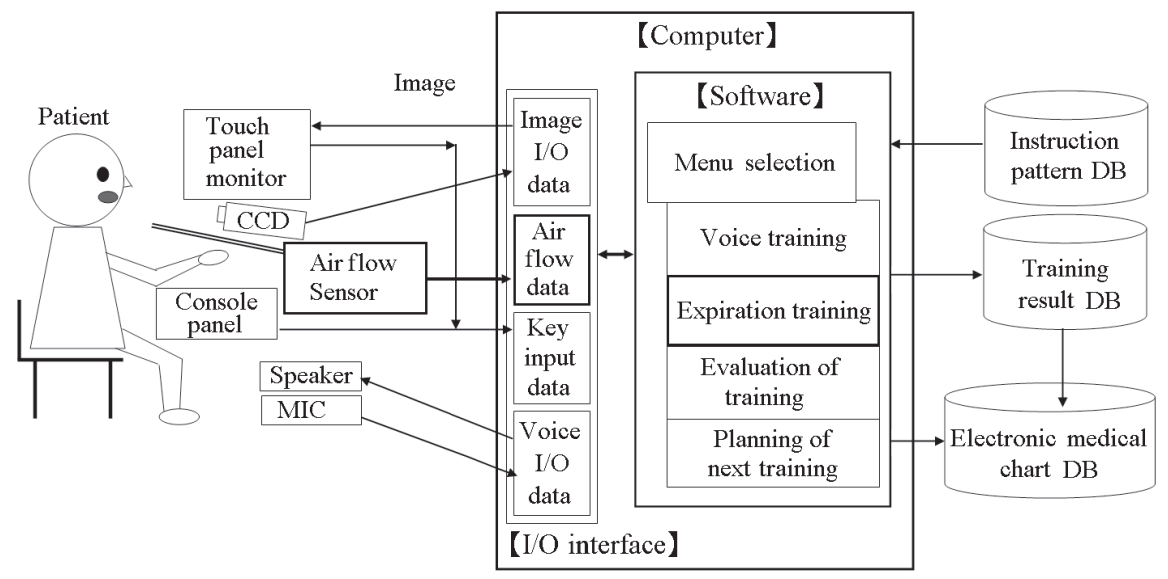

Fig. 1. Basic structure of training system. 
as CCD camera input, voice output, and mike input, among others, and can deal with various symptoms of people with speech, language and hearing disorders (i.e., trainees).

\subsection{Measurement of volume of expiratory flow}

The device for measuring the expiratory flow rate is shown in Fig. 2. The device consists of the following sensor units and an A/D converter. The data from the unit is fed into a computer.

- Air flow sensor 30-1000 (ml/s): FD-A1, Keyence

- Amplifier unit: FD-V40A, Keyence

- A/D converter 16 (bit) • 1MSPS: CSI-320416, Interface

In this system, the sampling period is set as $10(\mathrm{~ms})$ and the resolution of the $\mathrm{A} / \mathrm{D}$ converter is set at 12 (bit).

Figure 3 is a graph showing the expiration data of a healthy male adult while blowing rhythmically as measured using the air flow sensor.

\subsection{Example of expiration training}

The computer menu used in the expiration training system is shown in Fig. 4. As shown in Fig. 4, the training menu is set according to the following procedure:

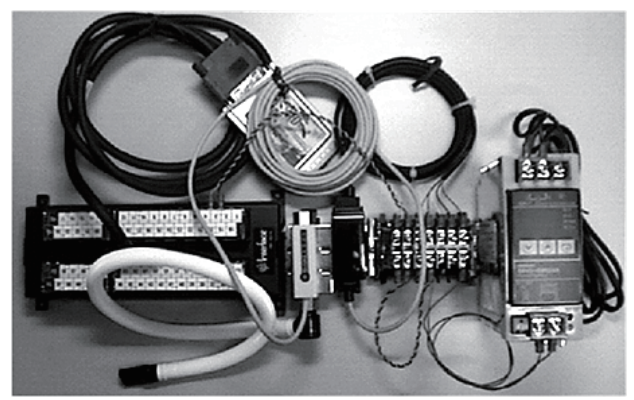

Fig. 2. Device for measuring expiratory flow rate in expiration training system.

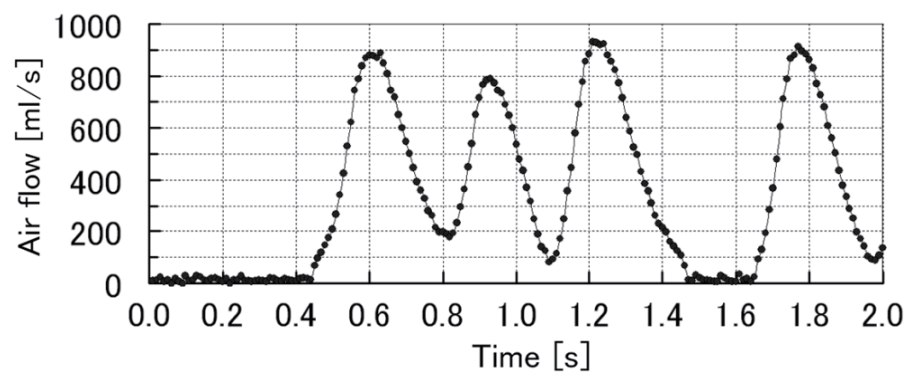

Fig. 3. Signal of airflow sensor. 
Setting

(1) Trainee

(2) Choose the word you want to train.

(3) Levels of training (Level 1, 2, or 3)

(4) Frequency of training

(5) Enter

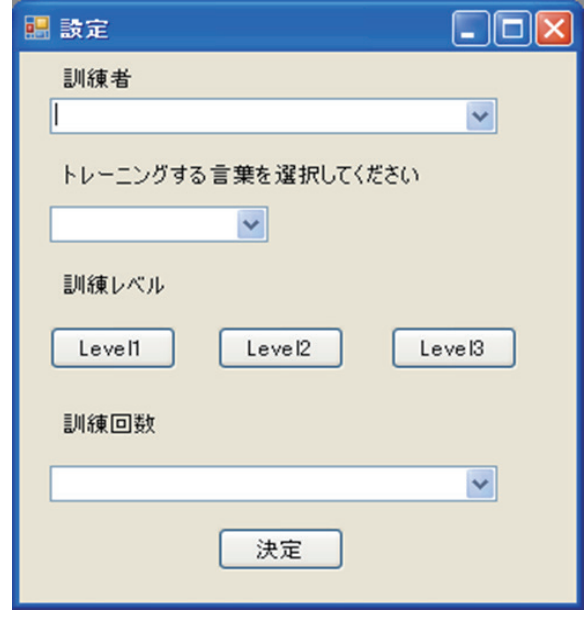

Fig. 4. (Color online) Top menu of training system.

(1) Select the trainee's number (select the ID and name of the trainee from the pull-down menu).

(2) Choose the shape of the mouth (e.g., "a" or "u") the trainee wants to train.

(3) Choose the level of training (the degree of difficulty increases in ascending order from Level 1 to Level 3).

(4) Select the number of times the trainee has received the training (automatic selection is possible).

(5) Click the "Enter" button and go to the training page.

The training page is shown in Fig. 5. The expiration pattern of the therapist is indicated in the upper part and that of the trainee in the lower part of the figure.

The strength of expiration is discretely expressed as zero, weak, medium, or strong. The model expiration is indicated as a simple pattern because when changes in the volume of expiratory flow are shown in a graph, such as that in Fig. 3, it is difficult for trainees to imitate the pattern. Therefore, a simple graph was adopted in this study.

In Fig. 5, the thresholds of a mouth shaped " $u$ " were set as 100, 400, and $700(\mathrm{ml} / \mathrm{s})$. When values measured by using the airflow sensor exceed the above thresholds, the level of expiratory flow is judged as weak, medium, or strong, respectively. These thresholds are adjusted on the basis of the trainees' condition, so that they can easily achieve the set goal, and motivation for training would increase.

Thresholds were determined by measuring the maximum expiratory flow $X_{\max }$ of the trainee before starting training. Strong was then defined as more than $70 \%$ of $X_{\max }$, medium as more than $40 \%$ of $X_{\max }$, and weak as $10 \%$ of $X_{\max }$ or more.

The therapist's expiration pattern is decided in advance, such that its length is $10 \mathrm{~s}$. During the training, the indicator (vertical line) moves from left to right with the course of time. Trainees control the timing, strength, and length of their breath by checking the graph and the indicator. 


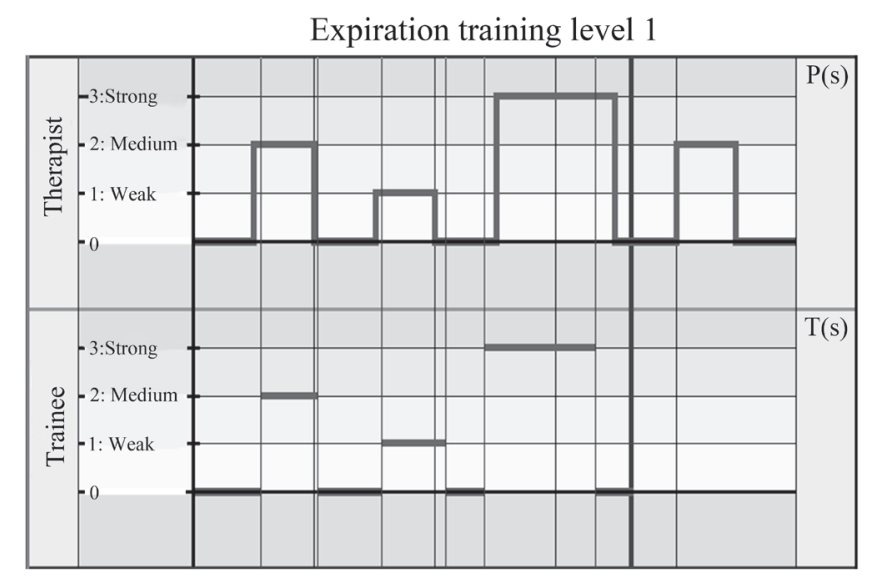

Fig. 5. Example of practice session.

As shown in Fig. 3, the performance characteristics of the sensor are such that there is an approximately $0.1 \mathrm{~s}$ delay at the start and finish of expiration. Therefore, assessment is not conducted during periods of sudden changes, such as at the start or end of expiration. The strength of the breath is assessed after sudden changes have ended.

\subsection{Expiration training menus}

Three levels of a therapist's expiration patterns are available, such that the trainees can choose an appropriate level.

As training advances, a therapist's expiration pattern becomes more refined with increases in the complexity of the interplay of changes in breath intensity and duration. A trainee proceeds to the next more complex level upon attaining advanced scores in the previous lower level of training.

The volume of expiratory flow differs on the basis of pronunciation using different mouth shapes. In this study, mouthpieces for the mouth shapes of "a" and " $u$ " were prepared. Trainees choose either one of these and put on the mouthpiece corresponding to the selected pronunciation.

\subsection{Evaluation of expiration training}

Evaluation was conducted by calculating the score based on the differences in expiration patterns between the therapist and the trainee. Lower scores indicated better results.

The score was calculated using eq. (1): $s=$ step time, $\{1, \ldots, 1000\} ; T(s)=$ strength of therapist's expiration, $\{0,1,2,3\} ; P(s)=$ strength of trainee's expiration, $\{0,1,2,3\}$.

$$
\text { Score }=\sum_{s=1}^{1000}|T(s)-P(s)|
$$


Other formulas used were as follows. First, the rate of concordance (\%) between the therapist's data and trainee's data was calculated using eq. (2).

$$
\text { Concordance rate }=\left(1-\frac{\text { Score }}{3000}\right) \times 100
$$

Next, the gap of timing expiration (difference in mean time of starting position in ms) was calculated using eq. (3): $n=$ number of times of therapist's expiration, $\{1,2,3, \ldots, N\}$; $T S(n)(\mathrm{ms})=$ initiation time of each $\mathrm{n}$ with therapist; $P S(n)(\mathrm{ms})=$ initiation time of each $n$ with trainee.

$$
\text { Difference in mean time of starting position }=\sum_{n=1}^{N}|T S(n)-P S(n)|
$$

The example shown in Fig. 5 indicates $N=4$.

Furthermore, the ability to control breath strength was calculated with the volume of expiratory flow $(\mathrm{ml} / \mathrm{s})$, which was measured using the sensor. Identical to eq. (1), $s$ $=$ step time, $\{1, \ldots, 1000\}$ and $T(s)=$ strength of therapist's expiration, $\{0,1,2,3\}$. The volume of expiratory flow of the trainee was expressed as $P F(s)=\{0-999\}$. Mean expiration levels that were over the target value (mean of over air flow $(\mathrm{ml} / \mathrm{s})$ ) and those that were under the value (mean of under air flow $(\mathrm{ml} / \mathrm{s})$ ) were calculated with eqs. (4) and (5). The counts $=\{1,2,3, \ldots\}$ were the frequencies of expiration levels that were over or under the target value among all the samples $(N=1000)$.

$$
\begin{gathered}
\text { Over air flow }=\sum_{s=1}^{1000}|T S(n)-P S(n)|(\text { if } T S(n)>P S(n), \text { Count } \leftarrow \text { Count }+1)( \\
\text { Mean of over air flow }=\frac{\text { Over air flow }}{\text { Count }}
\end{gathered}
$$

Regarding the mean of under air flow, (if $T S(n)>P S(n)$, Count $\leftarrow$ Count +1$)$ in eq. (4) was replaced by (if $T S(n)<P S(n)$, Count $\leftarrow$ Count +1 ).

The formulas shown above are useful for conducting evaluations based on different characteristics of trainees, by preparing various evaluation indexes. Results of calculations using eq. (1) are shown in the experimental results described below.

Furthermore, at each training session, not only the scores calculated with the formulas, but also the raw data of the expiratory flow were recorded, such that a future reevaluation would be possible. Recording with a time series facilitated confirming the effect of training.

\section{Experimental Methods}

Changes in training scores of healthy male adults $(N=5$, A-E) using Level 1 therapist's data shown in Fig. 5 are displayed in Fig. 6. Each trainee conducted 10 training sessions. 


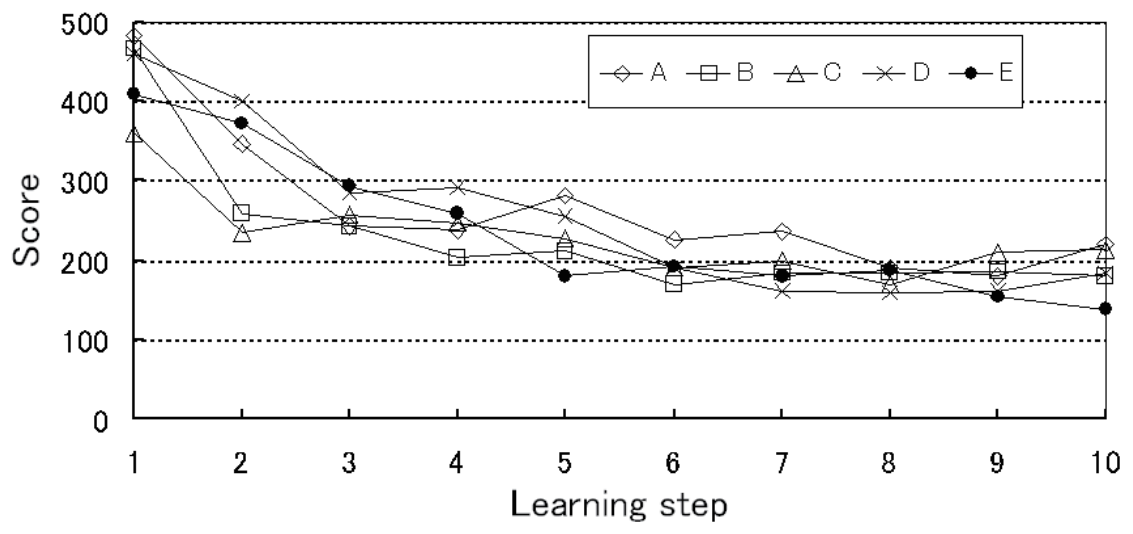

Fig. 6. Learning curve of expiration training.

Equations (2)-(5) were employed to derive scores on the vertical axis of Fig. 6. These scores represent differences between values presented by the therapist and values attained by the trainee with smaller scores representing improved results on the part of the trainee. As indicated in Fig. 6, improved scores serve to confirm progress through the step-wise training.

The feedback provided to the trainee is thought to better inform their adjustments in breath control (timing, strength, and length of expiration). In addition, successful reproduction of utterances requires repetitive training and the gamelike feedback in the form of a score may improve the level of engagement of the trainee.

\section{Conclusions}

The system developed in this study is a prototype and was tested with healthy adults. The results indicated an improvement of the score as the learning steps proceeded. Therefore, this system was considered effective for expiration training. However, longterm observations are necessary for confirming the improvement of expiration ability. It is suggested that in the future, this system should be tested and evaluated with people that actually require expiration training. Furthermore, adjustment of the airflow sensor (i.e., setting the thresholds for the strength of expiration) and the expiration pattern of therapists should be examined, so that trainees are able to more easily undergo the training.

Additionally, this system could be used as a method to confirm the rehabilitative effects of training for strengthening respiration muscles, such as "stretching to expand the mobility range of the lungs" and "training for strengthening muscles around the lungs", among others. Furthermore, the addition of test items assessing basic capacities, such as lung capacity and volume of expiratory flow, to training items suggested in this study (e.g., timing and control of the length and strength of blowing), would also facilitate the confirmation of the effects of rehabilitation. 
Recently, the aging population has become a serious problem in Japan, and maintaining the health of the elderly has become important. It is suggested that in addition to providing speech training for people with disabilities, this system could be used to address new needs, such as speech rehabilitation and anti-aging training for the elderly.

\section{Acknowledgements}

This work was supported by KAKENHI (Grant-in-Aid for Scientific Research (C) Nos. 21610007 and 24600005, Grant-in-Aid for JSPS Fellows No. 23-40189, and Research Activity Start-up No. 22830033).

\section{References}

1 J. R. Duffy: Motor Speech Disorders: Substrates, Differential Diagnosis, and Management, (C.V. Mosby, St. Louis, 1995).

2 K. M. Yorkston, D. R. Beukelman, E. A. Strand and K. R. Bell: Management of Motor Speech Disorders in Children and Adults, 2nd Ed. (C.V. Mosby, St. Louis, 1999).

3 N. Kurauchi (ed.): Gengo Chokaku Shogai Soron I (Kenpakusha, Tokyo, 2009).

4 K. Yamazaki (ed.): Gengo Chokaku Shogai Soron II (Kenpakusha, Tokyo, 2009).

5 Ed. M. Ito and S. Sasanuma: Manual for Speech-Language Therapy (Ishiyaku Publishers, Inc., Tokyo, 2002).

6 M. Nishio: Assessment of Motor Speech for dysarthria (Interuna Publishers, Inc., Tokyo, 2004).

7 Kotoba no Sutorecchi Kenkyukai (ed.): Kotoba no Sutorecchi Taiso (Meijitosho Shuppan Corp., Tokyo, 2004).

8 C. Ota and S. Kobayashi: Jpn. J. Spec. Educ. 16 (1979) 49 (in Japanese).

9 Y. Ogoshi, A. Nakai, Y. Mitsuhashi and C. Araki: Trans. IEEJ 130-C (2010) 458.

10 Y. Ogoshi, C. Araki, Y. Mitsuhashi and A. Nakai: Japan Patent No. 5360815 (2013).

\section{About the Authors}

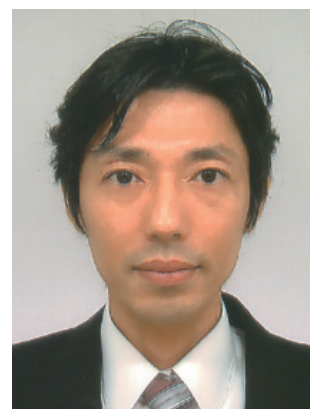

Yasuhiro Ogoshi is an associate professor of the Graduate School of Engineering, University of Fukui, Japan. He received his $\mathrm{Ph} . \mathrm{D}$. Degree in Engineering from the University of Kanazawa in 2001. He is engaged in studies related to human interface. 

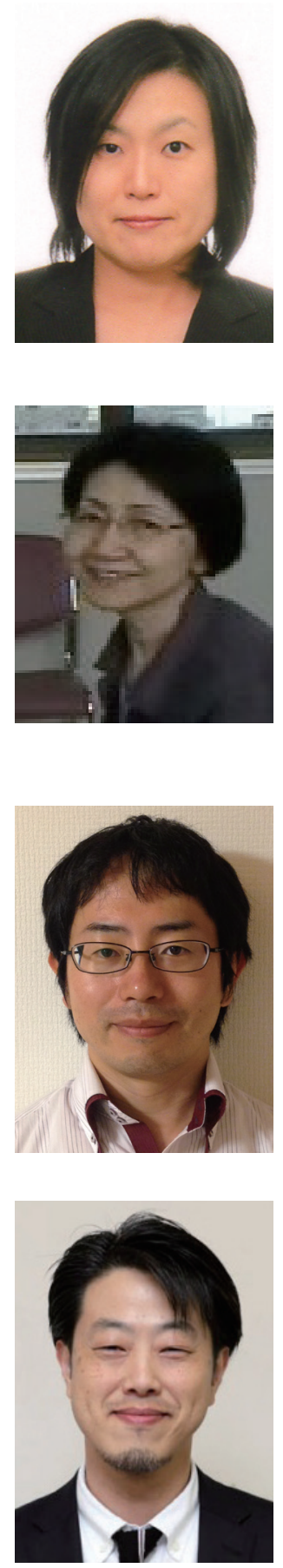

Sakiko Ogoshi is an assistant professor of the Career Design Laboratory for Gender Equality, Kanazawa University, Japan. She received her Ph.D. Degree in Engineering from the University of Kanazawa in 2004. She was a researcher at the Japan Society for the Promotion of Science and the Faculty of Education and Regional Studies, University of Fukui from 2011 to 2014. Her research interests include special needs and developmental disorders and knowledge management system applied information technology.

Kyoko Yamazaki is a professor of the Speech-Language-Hearing Therapy at the Department of Rehabilitation in Fukui College of Health Sciences. She completed her doctoral program without a doctoral degree in Special Education, Graduate School of Education, Tokyo University of Education in 1978. Since 1984, she has educated the students in the Speech-Language-Hearing Therapy Course, as well as counseled and cured the children with delayed development of language.

Tomohiro Takezawa received his Ph.D. Degree in Psychology from Hiroshima University. He specializes in cognitive abilities and assistive technology.

Akio Nakai, is currently the Senior Executive Vice Director of Hyogo Children's Sleep and Development Medical Research Center and a visiting professor of the Department of Child and Adolescent Psychological Medicine, University of Fukui Hospital, Japan. He received his M.D. in 1986 and Ph.D. in 1991 from Fukui Medical University. $\mathrm{He}$ is both a neurodevelopmental and behavioral pediatrician and a clinical developmental psychologist. He studied brain imaging of serotonin synthesis in McConnell Brain Imaging Centre, Montreal Neurological Institute, McGill University, Canada from 1998 to 2000. He is the Director of the Japanese Societies 
of Baby Science, the Japanese Society of Child Science, and the Japanese Society of Developmental Neuroscience. He is also the Editor-in-Chief of Baby Science and the Councilor of The Japanese Society of Child Neurology. He is working on several international collaborative studies of Developmental Coordination Disorder (DCD) with Canada, the Netherlands, Israel, UK, and Vietnam, as a representative of Japan in the International Society for Research into DCD.

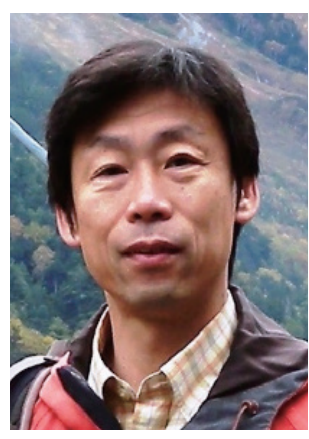

Kiyoharu Tsuji is a physical therapist of the Fukui Prefectural Rehabilitation Center for Children with Disabilities. He is affiliated with the Japanese Physical Therapy Association, Rehabilitation Engineering Society of Japan, Japanese Society on Severe Motor and Intellectual Disabilities, and Japan Sport Association for the Disabled. He specializes in neurological physical therapy, life support and environmental adjustment physical therapy, and sport training for the disabled.

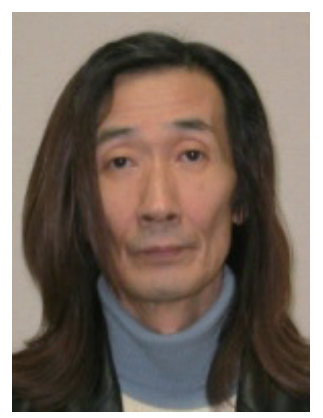

Yoshinori Mitsuhashi is a professor emeritus and currently a specially assigned professor of the Faculty of Education and Regional Studies at Fukui University, Japan. He graduated from Kwansei-Gakuin University, Department of Experimental Psychology in 1972, and finished his graduate study in 1980. He became an associate professor of Fukui University in 1981 and left as a professor in 2014. His research covers psychophysiology of cognition and education of handicapped children. In particular, he studies the cognitive process in the children and adults with neurodevelopmental disorders using the electrical activity of the brain. 